\title{
CARCINOMA IN RELATION TO PREgNANCY
}

\author{
By GLADYS HILL; M.A., M.D., F.R.C.S., F.R.C.O.G. \\ (Hon. Obst. Surg., Royal Free Hosp.; Asst. Obst., E.G.A. Hosp., etc.)
}

The consideration of this subject falls into two main categories:-

I. The effect of pregnancy upon the development of carcinoma.

2. The effect of pregnancy upon a developed carcinoma, whether the growth be in the reproductive system or elsewhere in the body.

The discussion on the effect of pregnancy upon the development of carcinoma is, however, $\stackrel{\triangleright}{\stackrel{D}{\Omega}}$ comparable to a house built without foundation, since, until the origin and causation of carci-ฆ noma is established upon a firm basis, any view which may be put forward must be, to a great $\overrightarrow{0}$ extent, speculative.

As to the origin of carcinoma, we know that such growths are commoner in organs which $\vec{\omega}$ are usually the site of rhythmical or cyclical physiological activity, such as the breasts, the alimentary canal, the uterus and the ovary. In the reproductive system, moreover, this physio-o logical activity is associated with marked and distinctive changes in the epithelial cells of the 3 . organ in question, a point well illustrated by the endometrium. We also know that a constant 0 predisposing factor is some local irritant, traumatic or chemical. This has been brought outiv by the work with mice and the experimental production of cancer by tar dermatitis.

There is evidence from the work of Gemmell and Jeffcote that the oestrogenic substances iv and the gonadotropic factors of the anterior pituitary and placenta promote epithelial hyper- $N$ plasia and metaplasia, and favour the development of breast carcinoma in rats and mice. The 9 essential physiological action of the ovarian and pituitary hormones is to stimulate growth. $\vec{c}$ The natural oestrogens belong to the hydrocarbons, as do the carcinogenic agents in tar, and are allied to the sterols. It has been observed that rats and mice of strains usually immune to carcinoma may develop it after treatment with the oestrogens. Emge believes, however, that $\vec{\ominus}$ the sex hormones promote carcinogenic change only when the cells they affect are geneticalig! conditioned to malignant change, and that therefore the action of these substances is secondany in importance and significance, to heredity. The work with the cancer retarding properties of ? the sex hormones of the opposite sex is suggestive. The fact that a measure of success has be en $\bar{\partial}$ achieved by this line of treatment lends support to the theory that hormones are among the aetiological factors in carcinogenesis. This raises the question of heredity in the developmentक of carcinoma, since all women with normal ovaries produce oestrogen, but not all develop $\overrightarrow{\vec{P}}$ carcinoma. The oestrogens may vary in quantity or quality, but there may well be hereditary응 differences in the tissues response or in the tumour inhibiting factors.

Finally, environment may have some influence on cancer growth. If this be true, unrelated women in the same environment would be more likely to be the subjects of carcinomatous growths of a similar character than related women in differing environments. It has been 3 widely observed, however, that women of the same stock, even when living and working under: diverse conditions, are far more alike in this respect than women who are unrelated.

As regards the origin of cancer in general, then, the following points emerge:-

I. The type of organ most commonly involved is one showing physiological rhythm.

2. The importance of local irritation, chemical or traumatic, has been proved.

3. The oestrogens and gonadotropic hormones cause tissue hyperplasia and metaplasia, and favour carcenogenesis.

4. Heredity plays a part in predisposing the individual to tumour development.

The question which must next engage our attention is: In which way, if any, does pregnancy N influence these factors?

The pregnant woman is the subject of an augmented metabolism. On the face of it this condition would be likely to coincide with an augmented rate of growth in any abnormal tissue present in the body. The pregnant woman produces gonadotropic hormones to a greater extent than the non-pregnant. Moreover, parturition itself is often a source of trauma which may@ provide a focus of local irritation, as may the changes in the lactating breast. It is of course 0 obvious that a carcinoma, whether genital or extra-genital, may develop during the course of ${ }_{0}^{\circ}$ pregnancy, but it is impossible to decide whether or not it would have occurred in the absence $\stackrel{\odot}{\circ}$ 
of pregnancy. A malignant growth may equally well occur in a woman who has borne children and in one who is barren, but it is well recognised that certain carcinomata do not occur, or are extremely rare, in nulliparae, e.g. the sphenoidal celled carcinoma of the lactating breast, and carcinoma of the cervix. Here are two examples where the connection between the pregnancy and the carcinoma is close, but both are growths in the reproductive system. There can be, then, no doubt that the liability of the individual to these forms of the disease is increased by pregnancy. Proliferation and down growths of cervical epethelium, especially during pregnancy, are believed to be due to the action of the oestrogenic and gonadtropic hormones present, and these may combine with local trauma to increase the liability to neoplastic change in this situation. A similar argument applies to breast carcinoma of the sphenoidal celled variety.

As regards extra-genital carcinoma, all that can usefully be said is that of the factors believed to be concerned in the origin of carcinoma, some at least, the sex hormones, are considerably increased during pregnancy. One illustration may be quoted here. The writer has seen two cases of malignant change in pigmented moles in patients during the early months of pregnancy. In neither case was there any history of repeated trauma.

A type of growth invariably linked with pregnancy is chorionic epithelioma, which may follow abortion, hydatidiform mole, or apparently normal parturition. In this instance alone have we definite evidence that the malignant neoplasm would not have occurred had the individual never been pregnant. The case of chorionic epithelioma falls into a class by itself, and the fact that there is a great increase in the production of anterior pituitary hormone in this condition lends support to the theory that there is a connection between the sex hormones and carcinogenesis. Of the aetiological factors concerned in cancer production it can be said that one, at least, the excess of gonadotropic hormone, is present to a marked degree in pregnancy.

The effect of pregnancy upon a developed carcinoma falls into two groups, an extra-genital carcinoma, and one of the reproductive system, the breast being included in this category.

\section{Developed Extra-genital Carcinoma and Pregnancy}

When a patient suffering from an extra-genital carcinoma becomes pregnant the general tendency is to advise that the pregnancy be terminated. It is obvious that treatment for the malignant condition, where treatment is possible, must be instituted immediately, and completed thoroughly at the expense of the pregnancy if necessary. But are there any grounds for thinking that the presence of the pregnancy, in itself, increases the risks of the patient? Here is the type of case where the only possible verdict is "Not Proven."

Superficially it would appear that the increased metabolism of pregnancy must accelerate the rate of growth and spread of the malignant lesion; moreover, if we accept, in any degree, the theory of the influence of the sex hormones, their presence in greater proportion would be unfavourable as regards the growth; finally, the physical strain of pregnancy and parturition might militate against the production of tumour inhibiting factors.

These arguments are specious enough, and are the more readily accepted since the grave risks to the patients of any malignant condition, even when the picture is entirely uncomplicated, are appreciated. It follows, therefore, that the pregnancy tends to be terminated in order to give the patient the best chance of recovery from the pathological lesion, and we are denied, for obvious and humane reasons, the opportunity of exact scientific observation. Even if the pregnancy be not deliberately removed, the treatment of the carcinoma may well bring about abortion when severe and extensive surgical procedure is involved.

Should the growth be an inoperable one, the pregnancy is still usually disregarded, since its continuance is thought to impose an unjustifiable strain on the patient. Only in the rare case of an individual refusing interference have we any opportunity of judging the relationship of the two conditions. On the strength of the admittedly scanty knowledge that we possess, however, it is reasonable to assume that the arguments put forward for the removal of the pregnancy are valid, and to agree that, as far as can be deduced, pregnancy has the effect of stimulating the growth of a developed extra-genital carcinoma.

\section{Developed Carcinoma of the Reproductive System and Pregnancy}

The two most important sites which have to be considered in this connection are the breasts and the cervix uteri.

Carcinoma of the body of the uterus does not occur in association with pregnancy, nor does 
carcinoma of the ovary. A lesion of a malignant nature on the vulva is also extremely rare. Equally, an advanced cervical neoplasm is incompatible with conception, but fertilisation may occur when a cervical carcinoma is in the initial stages, or when there is malignant change in an adenomatous polypus of the cervix. It is striking that in many of the cases of carcinoma of the cervix complicating pregnancy, which are recorded in literature, the malignant change originates in a polypus.

Speaking generally, it can be stated that when the neoplasm involves the reproductive system pregnancy causes a greatly increased rate of growth, and a much more extensive spread of the malignant process than would otherwise take place.

This is due to:-

I. the increased vascularity and activity of the organs.

2. the trauma of parturition and lactation.

3. the excessive production of gonadotropic hormones which inevitably affect the reproductive system to a greater extent than the other systems of the body.

As regards carcinoma of the breast, the physiological changes in this organ as a result of pregnancy accelerate this malignant process to a marked degree. Termination of the pregnancy would appear to be essential if there is to be any expectation of adequate and successful treatment. In this connection, the writer had an interesting experience.

A patient who had already borne several children was known to have a small slow-growing scirrhous $\mathbb{A}$ carcinoma of the breast, for which she persistently refused treatment. She became pregnant, but pur- iN posely avoided seeking medical advice or antenatal supervision because she was resolutely set against $\mathrm{N}$ any surgical measures.

In this pregnancy she was first seen at the 36 th week with the growth now ulcerated and fungating. Clinically the prognosis as regards the tumour appeared bad, if not hopeless. She went into labour within a few days, and was successfully delivered of a living child. After delivery there was a marked $\overleftarrow{c}$ and rapid improvement in the breast condition, but now that the disability from the growth had been severe, the patient consented to operative measures. When these were undertaken it was found possible to carry out a radical mastectomy. The patient made a good recovery, and a year later was in goo health. Here was an instance of a very slow-growing tumour which, nevertheless, advanced considerablyo and with much greater rapidity during the pregnancy. The patient's original aversion to treatment. made a very interesting observation possible.

Carcinoma of the cervix is a rare complication of pregnancy, the incidence being variously given, but is probably between one in 5,000 and one in to,000 cases of pregnancy.

Here again there can be little doubt that pregnancy brings about a marked and rapid deterioration in the local condition. Abortion is common, but probably only as a result of treatment. If undiagnosed, the growth spreads quickly and extensively; it also makes vaginal delivery impossible, as in such circumstances the cervix fails to dilate.

Two instances of a cervical carcinoma complicating advanced pregnancy have occurred in the personal experience of the writer in the last four years.

In the first case, a parous woman of 40 was admitted to hospital in labour. She stated that until the onset of labour she had not known that she was pregnant; vaginal bleeding, presumably from the neoplasm, had been mistaken for menstruation. A tentative diagnosis of placenta praevia had been made to account for the haemorrhage, but on examination the local condition was unmistakable. Vaginal delivery was impossible owing to the extent of the growth which had destroyed the cervix and infiltrated the upper part of the vagina. A Caesarean section was performed, and a living child delivered.

As there was no evidence of spread to the bases of the broad ligaments a panhysterectomy with removal of the appendages and as much vagina as possible was carried out at the same time. This was $D$ decided upon partly to obviate the risk of peritoneal infection, and partly to remove the neoplasm. The patient made a rapid and uneventful recovery from the operation, and was subsequently treated with radium. She was well for a year, but after that time there were signs of recurrence.

The second case was that of a multipara who complained of vaginal bleeding at the 26th week of pregnancy. She was admitted to hospital, and a cervical polypus the size of a Victoria plum was found to be the source of the bleeding. The polypus with its base was excised, and the pathological report $N_{\omega}$ showed it to be a simple adenoma. Her recovery was good, and the remainder of the pregnancy was $\sigma$ uneventful, with no further vaginal bleeding, or discharge. The patient, however, when admitted in labour looked ill and showed signs of obstruction. Examination revealed an extensive malignant growth 0 of the cervix. Delivery was effected by Caesarean section, but extension to the general peritoneum had $\overparen{\mathbb{D}}$ already occurred and was widespread. The patient died of malignant peritonitis within a short period. Here is an instance of a growth of the cervix, clinically and microscopically innocent at the 26 th week, which had become an advanced and widespread carcinoma fourteen weeks later, a rate of spread considerably more rapid than the average, and justifiably attributed to the presence of the pregnancy. 
There appear to be no figures to indicate the incidence of carcinoma, in general, in relation to pregnancy, but clinical experience suggests that it is, fortunately, low. On general principles it would appear that growth and spread are more rapid than the average when the neoplasm is associated with a cyesis, and in the case of carcinoma of the cervix there is some evidence to prove that this is so.

\title{
REFERENCES
}

GEMMELL and JEFFCOTE, J. Obstet. and Gynaec. B.E., 46, 985.

HOFFMANN, J. Obstet. and Gynaec. B.E., 46, 232.

EMGE, Western J. of Surg., 50, 32.

THURLOW, Western J. of Surg., 50, 439.

ALLEN, Endocrinology, 30, 942 .

\section{VOMITING IN THE NEW BORN}

\author{
By DAVID LEVI, M.S., F.R.C.S. \\ (Senior Surg. Infants' Hosp., Vincent Square)
}

I see vomiting in the newly-born from rather a specialised angle. Most of the children met with have been seen by a physician first, and such conditions as are purely medical have usually been treated. I propose to describe Hypertrophic Pyloric Stenosis, and then to recall some of the patients suffering from other conditions for whom my assistance has been sought.

Hypertrophic pyloric stenosis has become a widely recognised disease. Vomiting, the first symptom, usually occurs about the second to the fourth week, and it occurs in a previously healthy infant. At first, it is projectile. It occurs usually towards the end of a feed, or after the whole feed has been taken. The absence of the projectile nature of the vomit is of no great significance. If the food has remained in the stomach any length of time before it is vomited, the vomit is acid in re-action. The vomited material never contains bile. In an unsuccessfully treated or untreated child the vomited material changes in character, and may become dark coffee ground in appearance, and contain altered blood.

The first vomit may occur earlier in life than the second week, and two children stand out in my memory in this respect, one a seven-month premature infant, who vomited soon after birth, and upon whom I operated about fourteen days after birth to find a definite tumour. The second child lived in the North of England and was the third in its family. I had operated on its two elder brothers for hypertrophic pyloric stenosis, and on the third day after the birth of the third child the mother said it had hypertrophic pyloric stenosis as it behaved as its brothers had done. I opened its abdomen in spite of the fact that there was no palpable tumour. The stomach and pylorus appeared normal. I did nothing at all and closed the belly. The child gained half a pound, and then began to lose weight and vomit again. After three weeks, when the child had dropped one pound in weight, I re-opened it and found a definite pyloric tumour which was split in the usual way. The child then made an uninterrupted recovery.

These two observations suggest, firstly, that hypertrophy of the pylorus can occur in utero, and secondly, that there is a stage in the development of the disease before hypertrophy occurs, when a palpable tumour is not present. 